\title{
A Relativistic Version of the Ghirardi-Rimini-Weber Model
}

\author{
Roderich Tumulka*
}

August 16, 2006

\begin{abstract}
Carrying out a research program outlined by John S. Bell in 1987, we arrive at a relativistic version of the Ghirardi-Rimini-Weber (GRW) model of spontaneous wavefunction collapse. The GRW model was proposed as a solution of the measurement problem of quantum mechanics and involves a stochastic and nonlinear modification of the Schrödinger equation. It deviates very little from the Schrödinger equation for microscopic systems but efficiently suppresses, for macroscopic systems, superpositions of macroscopically different states. As suggested by Bell, we take the primitive ontology, or local beables, of our model to be a discrete set of space-time points, at which the collapses are centered. This set is random with distribution determined by the initial wavefunction. Our model is nonlocal and violates Bell's inequality though it does not make use of a preferred slicing of space-time or any other sort of synchronization of spacelike separated points. Like the GRW model, it reproduces the quantum probabilities in all cases presently testable, though it entails deviations from the quantum formalism that are in principle testable. Our model works in Minkowski space-time as well as in (well-behaved) curved background space-times.
\end{abstract}

PACS numbers: 03.65.Ta; 03.65.Ud; 03.30.+p. Key words: spontaneous wavefunction collapse; relativity; quantum theory without observers.

\section{Introduction}

... I am particularly struck by the fact that the [GRW] model is as Lorentz invariant as it could be in the nonrelativistic version. It takes away the ground of my fear that any exact formulation of quantum mechanics must conflict with fundamental Lorentz invariance. J. S. Bell [4]

*Dipartimento di Fisica dell'Università di Genova and INFN sezione di Genova, Via Dodecaneso 33 , 16146 Genova, Italy. E-mail: tumulka@mathematik.uni-muenchen.de 
In 1986, Ghirardi, Rimini, and Weber (GRW) proposed a model of spontaneous wavefunction collapse [19] based on a stochastic and nonlinear modification of the Schrödinger equation. When combined with a clear ontology, the GRW model turns quantum mechanics into a completely coherent theory. It resolves all paradoxes of quantum mechanics, in particular the measurement problem, and accounts for all phenomena of quantum mechanics in terms of an objective reality governed by mathematical laws. However, the GRW model is nonrelativistic.

In [4] and again in [6], John S. Bell emphasized that the GRW model has a propertymulti-time translation invariance - that can be regarded as a nonrelativistic surrogate of Lorentz invariance. This fact suggests that the biggest difficulty one would expect with turning a nonrelativistic theory into a relativistic one - the difficulty caused by the lack of a temporal ordering of spacelike separated events - is absent in the GRW model right from the start. We find this suggestion to be correct, and can indeed specify a relativistic version of the GRW model. We proceed along the lines of Bell's suggestions; in particular, we do not use a continuous spontaneous collapse model (corresponding to a diffusion process in Hilbert space and known as continuous spontaneous localization, or CSL), but rather a discrete one corresponding to a jump process in Hilbert space. Furthermore, we follow Bell in taking as the primitive ontology, or local beables, of the model the space-time points where the collapses are centered [4, 6, 21]. "A piece of matter then is a galaxy of such events" 44. We will call these points "flashes."

For a recent overview of spontaneous collapse models, see [3]. We regard our model as a step towards one possible explanation of the probability rules of quantum theory in the relativistic realm. This realm differs in two ways from that of nonrelativistic quantum mechanics, to which the GRW model applies: in the requirement of Lorentz symmetry, and in the phenomenon of particle creation and annihilation typical of quantum field theory. Here we will not be concerned with the latter, but focus on covariance, and thus on relativistic quantum mechanics; correspondingly, what we shall mean by "relativistic" is "Lorentz invariant," or its analogue in curved space-time.

Our relativistic model is surprisingly similar to the original GRW model, which it approaches in the nonrelativistic limit. Its structure is in no way more complicated than that of the GRW model in Bell's flash-based version. The two models have the following features in common: (i) the only objects in the universe (beyond the given space-time geometry) are the wavefunction and the flashes; (ii) two new constants of nature are needed, the collapse rate $1 / \tau$ per particle and the width $a$ of the localization; (iii) time reversal invariance is broken, while (in flat space-time) rotation, space translation, time translation, parity, and gauge invariance are obeyed; (iv) the dynamics is intrinsically stochastic.

Our model is based on relativistic quantum mechanics of $N$ particles. The question of identical particles we plan to address in a separate work; here we shall avoid this question and base our considerations on the quantum mechanics of distinguishable particles. We shall use the letter $i$ to denote (apart from $\sqrt{-1}$ ) the particle types, $i \in\{1, \ldots, N\}$, and $Q_{i}$ for the set of flashes belonging to $i$; the elements of this set are timelike separated from each other. 
The wavefunction is a multi-time wavefunction, i.e., it is defined on the Cartesian product of $N$ copies of space-time. We use the Dirac equation as the relativistic version of the Schrödinger equation determining the evolution of the wavefunction apart from the collapses (but we will mostly not worry whether the wavefunction lies in the positive energy subspace, except in Section 3.7). More precisely, we use the multi-time formalism with $N$ Dirac equations. For the consistency of this set of equations, we cannot have interaction potentials. To avoid discussing the question of interaction in relativistic quantum mechanics, we will assume non-interacting particles. Interaction can presumably be included by allowing for particle creation and annihilation, which however is beyond the scope of this paper. In any case, the difficulty of including interaction that we encounter here does not stem from the spontaneous collapses but rather from the mathematics of multi-time equations, and is thus encountered by every kind of relativistic quantum mechanics.

We will give three equivalent descriptions of our model. In one of them, we refer to an arbitrary slicing (foliation) of space-time into spacelike surfaces and obtain a Markov process for the temporal evolution (relative to this slicing) of wavefunction and flashes; since this picture is not manifestly covariant, we postpone it to the end. We will begin instead with an iterative construction of the flashes, and take this to be the definition of our model. In another description, we provide a formula for the joint distribution of the flashes on space-time in terms of the initial wavefunction.

This paper is organized as follows. In Section 2 we recall the definition of the GRW model. In Section 3 we define our relativistic variant. In Section 4 we compute joint distributions of the flashes and flash rates. In Section [5 we reformulate the model in terms of a temporal evolution relative to an arbitrary spacelike slicing of space-time. In Section [6] we show that the low velocity limit of our model is the GRW model. In Section 7 we discuss some predictions of our model. Finally, in Section 8 we conclude by comparing it to other models in the literature.

\section{The GRW Model}

\subsection{Definition}

We briefly recall the GRW model, following Bell's description 4]. The wavefunction $\Psi=\Psi\left(\boldsymbol{r}_{1}, \ldots, \boldsymbol{r}_{N}, t\right)$ evolves unitarily between the collapses. At the time $T$ when a flash of type $I \in\{1, \ldots, N\}$ occurs, at location $\boldsymbol{X} \in \mathbb{R}^{3}$, the wavefunction collapses according to

$$
\Psi\left(\boldsymbol{r}_{1}, \ldots, \boldsymbol{r}_{N}, T+\right)=\frac{j\left(\boldsymbol{r}_{I}-\boldsymbol{X}\right) \Psi\left(\boldsymbol{r}_{1}, \ldots, \boldsymbol{r}_{N}, T-\right)}{\rho_{I}^{1 / 2}(\boldsymbol{X}, T-)}
$$

where the jump factor is a Gaussian

$$
j(\boldsymbol{r})=K \exp \left(-\frac{\boldsymbol{r}^{2}}{2 a^{2}}\right)
$$


whose width $a$ is a new constant of nature, of order of magnitude $10^{-7} \mathrm{~m}$, and the normalization constant $K$ is chosen so that

$$
\int_{\mathbb{R}^{3}} d^{3} \boldsymbol{r}|j(\boldsymbol{r})|^{2}=1
$$

Furthermore,

$$
\rho_{i}(\boldsymbol{x}, t)=\int_{\mathbb{R}^{3 N}} d^{3} \boldsymbol{r}_{1} \cdots d^{3} \boldsymbol{r}_{N}\left|j\left(\boldsymbol{r}_{i}-\boldsymbol{x}\right) \Psi\left(\boldsymbol{r}_{1}, \ldots, \boldsymbol{r}_{N}, t\right)\right|^{2},
$$

so that the collapsed wavefunction in (II) is normalized. The rate for a collapse of type $i$ to occur in the volume element $d^{3} \boldsymbol{y}$ is

$$
\frac{1}{\tau} \rho_{i}(\boldsymbol{y}) d^{3} \boldsymbol{y}
$$

where $\tau$ is another new constant of nature, of order of magnitude $10^{15} \mathrm{sec}$. To put this differently,

$$
\operatorname{Prob}\left(Q_{i} \cap[t, t+d t] \times \mathbb{R}^{3}=\{Y\}, Y \in[t, t+d t] \times d^{3} \boldsymbol{y} \mid \Psi_{t}\right)=\frac{d t d^{3} \boldsymbol{y}}{\tau}\left\langle\Psi_{t}\left|\hat{\jmath}_{i}(\boldsymbol{y})^{2}\right| \Psi_{t}\right\rangle
$$

where $\hat{\jmath}_{i}(\boldsymbol{y})$ is the self-adjoint "collapse" operator that multiplies by the function $j\left(\boldsymbol{r}_{i}-\right.$ $\boldsymbol{y})$, and $Q_{i}$ is the set of all flashes with label $i$ (a subset of space-time).

\subsection{Another Definition}

We now give a second, equivalent, formulation of the GRW model that is closer to the way we will formulate the definition of our relativistic model. A first change consists in that, rather than having the wavefunction change discontinuously at some time $T$, we will speak of two different wavefunctions, one representing the situation before collapse and the other the situation after (or better, one uncollapsed and one collapsed), and extend both wavefunctions to all times, future and past, using the unitary, collapse-free evolution. Hence, in this terminology and notation, wavefunctions never collapse. If that seems paradoxical, we emphasize that the goal is to define the distribution of the random sets $Q_{i}$, as it is only the flashes that chairs, tables, and observers are made of, and whatever mathematical formulation leads to the right distribution is allowable.

Assume, as we will do in the relativistic model, that the $N$ particles do not interact, that is, that the Hamiltonian is of the form $H=H_{1}+\ldots+H_{N}$ where $H_{i}$ acts only on the $i$-th coordinate of the wavefunction-so that $H_{i}$ commutes with $H_{j}, i \neq j$. This allows us to define the Schrödinger evolution also for a multi-time wavefunction

$$
\Psi\left(\boldsymbol{r}_{1}, t_{1}, \ldots, \boldsymbol{r}_{N}, t_{N}\right)=e^{-i H_{1} t_{1} / \hbar} \cdots e^{-i H_{N} t_{N} / \hbar} \Psi\left(\boldsymbol{r}_{1}, 0, \ldots, \boldsymbol{r}_{N}, 0\right) .
$$

The building block of the reformulation of the GRW model is the following procedure for obtaining, from given time values $T_{1}, \ldots, T_{N}$ (later taken to be times of flashes) and 
a given wavefunction $\Psi$ on $\mathbb{R}^{4 N}$, new random time values $T_{1}^{\prime}, \ldots, T_{N}^{\prime}$ (later taken to be times of subsequent flashes), associated random locations $\boldsymbol{Y}_{1}, \ldots, \boldsymbol{Y}_{N} \in \mathbb{R}^{3}$, and a new (collapsed) wavefunction $\Phi$ on $\mathbb{R}^{4 N}$ : Let $\Delta T_{1}, \ldots, \Delta T_{N}$ be independent, exponentially distributed random variables with expectation $\tau$, and set $T_{i}^{\prime}=T_{i}+\Delta T_{i}$. The joint distribution of the $\boldsymbol{Y}_{i}$,

$$
\operatorname{Prob}\left(\boldsymbol{Y}_{1} \in d^{3} \boldsymbol{y}_{1}, \ldots, \boldsymbol{Y}_{N} \in d^{3} \boldsymbol{y}_{N}\right)=\rho\left(\boldsymbol{y}_{1}, \ldots, \boldsymbol{y}_{N}\right) d^{3} \boldsymbol{y}_{1} \cdots d^{3} \boldsymbol{y}_{N}
$$

has density $\rho: \mathbb{R}^{3 N} \rightarrow \mathbb{R}$ defined by

$$
\rho\left(\boldsymbol{y}_{1}, \ldots, \boldsymbol{y}_{N}\right)=\int_{\mathbb{R}^{3 N}} d^{3} \boldsymbol{z}_{1} \cdots d^{3} \boldsymbol{z}_{N}\left|j\left(\boldsymbol{z}_{1}-\boldsymbol{y}_{1}\right) \cdots j\left(\boldsymbol{z}_{N}-\boldsymbol{y}_{N}\right) \Psi\left(\boldsymbol{z}_{1}, T_{1}^{\prime}, \ldots, \boldsymbol{z}_{N}, T_{N}^{\prime}\right)\right|^{2}
$$

Now define, for all $\left(\boldsymbol{z}_{1}, \ldots, \boldsymbol{z}_{N}\right) \in \mathbb{R}^{3 N}$,

$$
\Phi\left(\boldsymbol{z}_{1}, T_{1}^{\prime}, \ldots, \boldsymbol{z}_{N}, T_{N}^{\prime}\right)=\frac{j\left(\boldsymbol{z}_{1}-\boldsymbol{Y}_{1}\right) \cdots j\left(\boldsymbol{z}_{N}-\boldsymbol{Y}_{N}\right) \Psi\left(\boldsymbol{z}_{1}, T_{1}^{\prime}, \ldots, \boldsymbol{z}_{N}, T_{N}^{\prime}\right)}{\rho^{1 / 2}\left(\boldsymbol{Y}_{1}, \ldots, \boldsymbol{Y}_{N}\right)}
$$

and extend $\Phi$ to all other times values by unitary multi-time evolution such as (7).

The random sets $Q_{i}$ of flashes are obtained by iterating this procedure. We start with $T_{1}=0, \ldots, T_{N}=0$ and the initial wavefunction, obtain the first flash $\left(T_{i}^{\prime}, \boldsymbol{Y}_{i}\right)$ in every $Q_{i}$, and then take the times of these flashes and the new wavefunction as the input of the next round of the procedure. It can be shown that this definition is equivalent to the definition given in the previous subsection as it leads to the same random sets $Q_{i}$. We also obtain in this way a sequence of wave functions, each on $\mathbb{R}^{4 N}$, each a solution to (77), and each associated with a set of $N$ flashes.

\section{The Relativistic Model}

In this section we define the relativistic model. We will use a rather abstract formulation that has the advantages of being concise, simple, manifestly consistent, and manifestly covariant.

\subsection{The Dirac Equation}

We begin with recalling some relevant aspects of the Dirac equation and introducing some notation along the way. We generally use the letters $x, y, \ldots, X, Y, \ldots$ to denote space-time points, where capital letters usually stand for random space-time points.

As the particles are not interacting, our model does not require that they live in the same space-time; instead, we may have $N$ space-time manifolds $\mathscr{M}_{1}, \ldots, \mathscr{M}_{N}$ (which may be flat or curved), such that the wavefunction is a function on $\mathscr{M}_{1} \times \cdots \times \mathscr{M}_{N}=\prod_{i} \mathscr{M}_{i}$, and the set $Q_{i}$ of flashes of type $i$ is a discrete subset of $\mathscr{M}_{i}$. We find that this greater mathematical generality, though physically unnecessary, facilitates the mathematical treatment of the model. 
The evolution of the wavefunction $\Psi=\Psi\left(x_{1}, \ldots, x_{N}\right)$ is determined, apart from the collapses, by the Dirac equation

$$
i \hbar \gamma_{i}^{\mu}\left(\nabla_{i, \mu}-\frac{i e_{i}}{\hbar} A_{i, \mu}\left(x_{i}\right)\right) \Psi=m_{i} \Psi
$$

Here, $m_{i}$ and $e_{i}$ are mass and charge of particle $i, \nabla_{i}$ is the (covariant) derivative on $\mathscr{M}_{i}$, and $A_{i}$ the electromagnetic vector potential on $\mathscr{M}_{i}$. $\Psi$ takes values in $\left(\mathbb{C}^{4}\right)^{\otimes N}$ or, in the case of curved space-times, is a cross-section of the vector bundle

$$
\mathscr{D}=\bigcup_{x_{1} \in \mathscr{M}_{1}, \ldots, x_{N} \in \mathscr{M}_{N}} \mathscr{D}_{\left(x_{1}, \ldots, x_{N}\right)}=\bigcup_{x_{1} \in \mathscr{M}_{1}, \ldots, x_{N} \in \mathscr{M}_{N}} \mathscr{D}_{1, x_{1}} \otimes \cdots \otimes \mathscr{D}_{N, x_{N}},
$$

where $\mathscr{D}_{i}$ is the bundle of Dirac spin-spaces associated with $\mathscr{M}_{i}$.

As in Section 2.2, we will, throughout Section 3, take the wavefunctions to always obey the collapse-free evolution, this time given by the multi-time Dirac equation (11), and to be defined on all of $\prod_{i} \mathscr{M}_{i}$. In particular, at any collapse we will consider two wavefunctions rather than one that changes discontinuously.

A remark is necessary on consistency of the multi-time formalism. Multi-time equations are not always consistent; what can go wrong is that the propagator for the $i$-th time coordinate (relative to some coordinate system) fails to commute with the $j$-th propagator, $j \neq i$, in which case it is impossible to find, for every initial wavefunction, a wavefunction on $\prod_{i} \mathscr{M}_{i}$ solving all $N$ evolution equations simultaneously. The set of equations (11), however, is consistent because the metric and vector potential acting on the coordinate $x_{i}$ do not depend on the other coordinates $x_{j}, j \neq i$.

We now briefly explain how the Dirac equation (11) defines unitary propagators on suitable Hilbert spaces. Consider first the one-particle Dirac equation

$$
i \hbar \gamma^{\mu}\left(\nabla_{\mu}-\frac{i e}{\hbar} A_{\mu}\right) \Psi=m \Psi .
$$

We need and assume from now on that there are no closed timelike curves. With every spacelike surface $\Sigma$ is associated a Hilbert space $L^{2}(\Sigma)$, and with any two spacelike Cauchy ${ }^{1}$ surfaces $\Sigma$ and $\Sigma^{\prime}$ is associated a unitary propagator $\hat{U}_{\Sigma}^{\Sigma^{\prime}}: L^{2}(\Sigma) \rightarrow L^{2}\left(\Sigma^{\prime}\right)$ as follows. $L^{2}(\Sigma)$ contains cross-sections of the bundle $\mathscr{D}$ of Dirac spin spaces (which on Minkowski space-time are just $\mathbb{C}^{4}$ ) restricted to $\Sigma$ and is endowed with the scalar product

$$
\langle\Psi \mid \Phi\rangle=\int_{\Sigma} d^{3} x \bar{\Psi}(x) n_{\mu}(x) \gamma^{\mu} \Phi(x)
$$

where $n_{\mu}(x)$ is the (future-directed) unit normal vector on $\Sigma$ at $x$, and the volume measure $d^{3} x$ is the one arising from the Riemann metric on $\Sigma$. For simplicity, we will often write

$$
|\Psi(x)|^{2}=\bar{\Psi}(x) n_{\mu}(x) \gamma^{\mu} \Psi(x)
$$

when it is clear which surface $\Sigma$ we are considering. Unitarity of the propagator $\hat{U}_{\Sigma}^{\Sigma^{\prime}}$ follows from the continuity equation

$$
\nabla_{\mu}\left(\bar{\Psi} \gamma^{\mu} \Psi\right)=0
$$

\footnotetext{
${ }^{1}$ A Cauchy surface is a surface intersected by every complete timelike curve.
} 
which is a consequence of (13). Of course, $\hat{U}_{\Sigma^{\prime}}^{\Sigma^{\prime \prime}} \hat{U}_{\Sigma}^{\Sigma^{\prime}}=\hat{U}_{\Sigma}^{\Sigma^{\prime \prime}}$, and $\hat{U}_{\Sigma}^{\Sigma}$ is the identity operator on $L^{2}(\Sigma)$. For multi-time wavefunctions, we will only consider spacelike surfaces of product form, $\Sigma_{1} \times \cdots \times \Sigma_{N}$. Then, the associated Hilbert space coincides with the tensor product of the Hilbert spaces associated with the $\Sigma_{i}$, and the unitary propagator between two such spaces coincides with the tensor product of the unitary propagators for each $i$.

\subsection{Notation}

We need some more notation. Let $\mathscr{F}(x)$ denote the future of $x$, i.e., the future light cone and its interior including $x$ itself, and $\mathscr{P}(x)$ the past of $x$. When $S$ is a subset of space-time we write $\mathscr{F}(S)$ ("the future of $S$ ") for $\bigcup_{x \in S} \mathscr{F}(x)$ and $\mathscr{P}(S)$ ("the past of $\left.S^{\prime \prime}\right)$ for $\bigcup_{x \in S} \mathscr{P}(x)$. For $y \in \mathscr{F}(x)$, let t-dist $(y, x)$ be the timelike distance of $y$ from $x$, i.e., the supremum of the lengths of all timelike curves connecting $x$ to $y$; for Minkowski space-time,

$$
\operatorname{t}-\operatorname{dist}(y, x)=\left(\left(y^{\mu}-x^{\mu}\right)\left(y_{\mu}-x_{\mu}\right)\right)^{1 / 2} \text {. }
$$

For $r \geq 0$, let

$$
\mathscr{H}_{r}(x)=\{y \in \mathscr{F}(x): \mathrm{t}-\operatorname{dist}(y, x)=r\}
$$

be the future surface of timelike distance $r$ from $x$, or the $r$-hyperboloid; for $r=0$ this is the future light cone. For $y \in \mathscr{F}(x)$, we also write $\mathscr{H}(y, x)=\mathscr{H}_{\mathrm{t} \text {-dist }(y, x)}(x)$ for the hyperboloid centered at $x$ containing $y$. If $\Sigma$ is a spacelike surface and $x, y \in \Sigma$, we write $\operatorname{s-dist}_{\Sigma}(x, y)$ for the spacelike distance from $x$ to $y$ along $\Sigma$, i.e., the infimum of the Riemannian lengths of all curves in $\Sigma$ connecting $x$ to $y$. The speed of light is denoted by $c$.

\subsection{Law of the Flashes}

We are now prepared to write down the stochastic law of the flashes. The building block is the following procedure for obtaining, from given flashes $X_{1}, \ldots, X_{N}$ and a (normalized) wavefunction $\Psi$ obeying (11), new flashes $Y_{1}, \ldots, Y_{N}$ and a new wavefunction $\Phi$ obeying (11).

Let $\Delta T_{1}, \ldots, \Delta T_{N}$ be independent, exponentially distributed random variables with expectation $\tau$. Choose $\left(Y_{1}, \ldots, Y_{N}\right)$ at random from $\Sigma_{1} \times \cdots \times \Sigma_{N}=\prod_{i} \Sigma_{i}$, where $\Sigma_{i}=\mathscr{H}_{c \Delta T_{i}}\left(X_{i}\right)$, with distribution

$$
\operatorname{Prob}\left(Y_{1} \in d^{3} y_{1}, \ldots, Y_{N} \in d^{3} y_{N}\right)=\rho\left(y_{1}, \ldots, y_{N}\right) d^{3} y_{1} \cdots d^{3} y_{N}
$$

as follows. The volume of $d^{3} y_{i}$ is computed using the Riemann metric on $\Sigma_{i}$; the distribution density $\rho: \prod_{i} \Sigma_{i} \rightarrow \mathbb{R}$ is defined by

$$
\rho\left(y_{1}, \ldots, y_{N}\right)=\int_{\prod_{i} \Sigma_{i}} d^{3} z_{1} \cdots d^{3} z_{N}\left|j_{\Sigma_{1}}\left(y_{1}, z_{1}\right) \cdots j_{\Sigma_{N}}\left(y_{N}, z_{N}\right) \Psi\left(z_{1}, \ldots, z_{N}\right)\right|^{2}
$$


and, for any spacelike surface $\Sigma$, the jump factor $j_{\Sigma}: \Sigma \times \Sigma \rightarrow \mathbb{R}$ is defined by

$$
j_{\Sigma}(y, z)=K_{\Sigma}(z) \exp \left(-\frac{\mathrm{s}^{-\operatorname{dist}_{\Sigma}^{2}(y, z)}}{2 a^{2}}\right)
$$

with $K_{\Sigma}(z)$ chosen so that

$$
\int_{\Sigma} d^{3} y\left|j_{\Sigma}(y, z)\right|^{2}=1
$$

Now define $\Phi$ on $\prod_{i} \Sigma_{i}$ by

$$
\Phi\left(z_{1}, \ldots, z_{N}\right)=\frac{j_{\Sigma_{1}}\left(Y_{1}, z_{1}\right) \cdots j_{\Sigma_{N}}\left(Y_{N}, z_{N}\right) \Psi\left(z_{1}, \ldots, z_{N}\right)}{\rho^{1 / 2}\left(Y_{1}, \ldots, Y_{N}\right)}
$$

and define it on the remainder of $\prod_{i} \mathscr{M}_{i}$ by extending via (11).

To the extent that the hyperboloids $\Sigma_{i}=\mathscr{H}_{c \Delta T_{i}}\left(X_{i}\right)$ are Cauchy surfaces (see remarks in the subsequent subsection), $\Phi$ is uniquely determined on $\prod_{i} \mathscr{M}_{i}$ from initial data on $\prod_{i} \Sigma_{i}$ (and normalized due to (20) $), \Psi$ is normalized on $\prod_{i} \Sigma_{i}$, and, by (22), $\rho$ is normalized in the sense

$$
\int_{\prod_{i} \Sigma_{i}} d^{3} y_{1} \cdots d^{3} y_{N} \rho\left(y_{1}, \ldots, y_{N}\right)=1
$$

Note also that $Y_{i} \in \mathscr{F}\left(X_{i}\right)$.

Our relativistic model is defined by iterating this procedure. As initial data, specify a wavefunction $\Psi=\Psi^{0}$ and one flash $X_{i}^{0}$ of every type $i$. Apply the above procedure to obtain $\Psi^{1}=\Phi$ and a new flash $X_{i}^{1}=Y_{i}$ of every type. Repeat the procedure with $\Psi=\Psi^{1}$ and $X_{i}=X_{i}^{1}$, and so on. In this way, obtain for every type $i$ a random sequence of flashes, $Q_{i}=\left\{X_{i}^{0}, X_{i}^{1}, X_{i}^{2}, \ldots\right\}$.

Concerning the initial flashes of the universe, it seems an idea worth considering that the Big Bang, i.e., the initial singularity of the space-time geometry, is the space-time location of the initial flash for each $i \in\{1, \ldots, N\}$. This could have the status of a law, and would remove the arbitrariness of the initial flashes. Of course, the mathematics of the model works with any choice of the initial flashes.

\subsection{Hyperboloids and Cauchy Surfaces}

A hyperboloid need not be a Cauchy surface in $\mathscr{M}_{i}$; in fact, it never is in Minkowski space-time, ${ }^{2}$ though it is "almost Cauchy" in the sense that most complete timelike curves do intersect a given hyperboloid: the ones avoiding the hyperboloid accelerate to the speed of light. However, it is not necessary for our purposes that the hyperboloids be Cauchy surfaces. It is sufficient that, for a single particle,

the Dirac equation defines a unitary evolution operator

$U_{\Sigma}^{\mathscr{H}}: L^{2}(\Sigma) \rightarrow L^{2}(\mathscr{H})$ for every Cauchy surface $\Sigma$ and every hyperboloid $\mathscr{H}$.

\footnotetext{
${ }^{2}$ To see this, consider the following example of a complete timelike curve $x(t)$ that does not intersect the unit hyperboloid $\mathscr{H}_{1}(0,0,0,0)$ : the uniformly accelerated curve $x(t)=\left(c t, 0,0, \sqrt{1+c^{2} t^{2}}\right)$.
} 
We conjecture, but do not have a proof, that this is the case in Minkowski space-time for a large class of vector potentials $A_{\mu}$. We hope to be able to provide a proof in a future work. Here is a partial argument: To show that the evolution is unitary requires to show that it is onto and norm-preserving. To show the latter it should suffice to show that, for all wavefunctions $\psi$ from a dense subspace of $L^{2}(\Sigma)$, no more than a set of measure zero of flow lines of the $|\psi|^{2}$ distribution (Bohmian trajectories) avoid the hyperboloid. This presumably amounts to the vanishing of the probability flux into the future null infinity. This could be expected to be the case for $\psi$ from the form domain of the Hamiltonian, because to accelerate a positive amount of Bohmian trajectories to the speed of light should require, in some sense, an infinite amount of energy, while such $\psi$ has finite energy expectation. Indeed this conclusion apparently follows, for static electromagnetic fields tending to zero fast enough at infinity and for a suitable class of $\psi$ 's, from the flux-across-surfaces theorem [17] using the global existence of Bohmian trajectories 28].

How about other space-times than Minkowski? In some space-times, hyperboloids actually are Cauchy surfaces and so the problem is absent; for example, think of Minkowski space-time modulo a spacelike 3-lattice. Relevant conditions for this case may be emptiness of the future null infinity and absence of future singularities. The future null infinity could be expected to be empty when "space has finite volume growing not too quickly," such as in Minkowski space modulo a spacelike 3-lattice. In space-times in which, as in Minkowski space-time, hyperboloids are not Cauchy surfaces, a relevant condition for (25) seems to be that, for all $\psi$ from a dense subspace, the probability flux into the future null infinity vanishes.

For particles with zero rest mass, such as photons and gravitons, one would expect that there typically is a positive probability flux into the future null infinity. ${ }^{3}$ One way of avoiding this problem would be to postulate that flashes are associated only with massive particles, and to have the arguments of the wavefunction corresponding to massless particles get integrated out in (20) along an arbitrary Cauchy surface.

\subsection{Nonlocality}

The model is nonlocal. This is manifest in (19) in that the joint distribution of the flashes $Y_{i}$ and $Y_{j}, j \neq i$, does not factorize. One can easily find situations in which the events $Y_{i}$ and $Y_{j}$ (now taken to lie in the same space-time manifold) are spacelike separated, and still the distribution of $Y_{i}$ depends on the realization of $Y_{j}$; or, of course, we may view it the other way round: that the distribution of $Y_{j}$ depends on the realization of $Y_{i}$.

\subsection{Generations}

Since the flashes were constructed here together in generations, i.e., groups $Y_{1}, \ldots, Y_{N}$ of $N$ flashes, one may wonder whether the theory presupposes, or provides, some structure

\footnotetext{
${ }^{3}$ This remark has been kindly pointed out to me by Fay Dowker.
} 
beyond the mere flashes and their labels, a structure defining which flashes belong to the same generation. Such a structure would seem against the spirit of relativity, as much perhaps as a preferred slicing of space-time into hypersurfaces, since it would define, for two spacelike separated flashes, which is earlier and which is later, if they belong to different generations.

In fact, however, the theory neither presupposes nor provides a grouping of the flashes into generations. Rather, one could take as the initial flashes a combination of some of the first-generation flashes $X_{i}$ and some of the second-generation flashes $Y_{i}$, and obtain the same (conditional) distribution of the future flashes. This will become manifest in the reformulation of the law of the flashes of Section 4.1 .

\subsection{Negative Energy Contributions}

In the context of the Dirac equation, one often considers wavefunctions with negative energy as unphysical, more precisely those wavefunctions containing, for at least one particle, at least some contribution from the negative-energy subspace of the 1-particle Dirac Hamiltonian. Such wavefunctions we will call "non-positive" for short, and the other wavefunctions, containing exclusively positive-energy contributions, "positive." The collapse accompanying a flash will generically map a positive wavefunction to a non-positive one. This fact poses a problem if we want to take the model seriously, and in particular if we want to extend it to quantum field theories incorporating antiparticles.

One might conclude that the law for collapsing the wave function should be so modified that the collapsed wavefunction remains positive, for example by simply projecting the wavefunction, on top of the usual multiplication by a Gaussian as in (23), to the space of positive wavefunctions. In formulas, if $P_{+}$denotes this projection, and if we abbreviate the collapse law (23) as

$$
\Phi \propto j \Psi
$$

the modified collapse law corresponds to

$$
\Phi \propto P_{+}(j \Psi) .
$$

Since $P_{+}$is the same operator as convolution with a suitable function whose width is small (of the order of magnitude of the Compton wavelength), and thus does not change the $|\Phi|^{2}$ distribution a lot, replacement of (23) with (26) would perhaps entail merely a small change in the distribution of the flashes.

Alternatively, one might conclude that the spontaneous collapses can lead to spontaneous pair creation, since non-positive wavefunctions would appear to have something to do with anti-particle states. This effect might entail observable deviations from quantum mechanics. For calculating concrete predictions, it would seem necessary to first precisely formulate a version of the model that incorporates particle creation and uses wavefunctions from Fock space, but one might guess already from the present model that the probability of pair production at a collapse could be of the order of magnitude of $\left\|\left(1-P_{+}\right)(j \Psi)\right\|^{2} /\|j \Psi\|^{2}$. 


\section{Distribution Formulas}

In the previous section we have defined, in a rather abstract way, the distribution of the flashes in space-time. We will find it helpful to have further expressions for this distribution; such expressions we provide in this section.

\subsection{The First $n$ Flashes}

It is now straightforward, though somewhat tedious, to derive an explicit formula for the joint distribution of the first $n_{i}$ flashes of type $i$, given the "initial" flashes $X_{1}^{0}, \ldots, X_{N}^{0}$. For any choice of spacelike (Cauchy) surfaces $\Sigma_{i}^{0}$, we can express the distribution in terms of the initial wavefunction $\Psi^{0}$, restricted to the Cartesian product of the $\Sigma_{i}^{0}$, in the form

$$
\operatorname{Prob}\left(X_{i}^{k} \in d^{4} x_{i}^{k} ; i=1, \ldots, N ; k=1, \ldots, n_{i}\right)=\left\langle\Psi^{0}\left|E^{(\vec{n})}\left(\prod_{i=1}^{N} \prod_{k=1}^{n_{i}} d^{4} x_{i}^{k}\right)\right| \Psi^{0}\right\rangle,
$$

where the scalar product is taken in $\otimes_{i} L^{2}\left(\Sigma_{i}^{0}\right)$. Here, $\vec{n}=\left(n_{1}, \ldots, n_{N}\right)$, and $E^{(\vec{n})}$ is a positive-operator-valued measure (POVM) on $\prod_{i} \mathscr{M}_{i}^{n_{i}}$. It is of a product form,

$$
E^{(\vec{n})}\left(\prod_{i=1}^{N} \prod_{k=1}^{n_{i}} d^{4} x_{i}^{k}\right)=\bigotimes_{i=1}^{N} E_{i, X_{i}^{0}}^{\left(n_{i}\right)}\left(\prod_{k=1}^{n_{i}} d^{4} x_{i}^{k}\right)
$$

where $E_{i}^{(n)}=E_{i, x}^{(n)}$ is a POVM on $\mathscr{M}_{i}^{n}$, defined on $L^{2}\left(\Sigma_{i}^{0}\right)$ by

$$
\begin{aligned}
& E_{i, x_{i}^{0}}^{(n)}\left(\prod_{k=1}^{n} d^{4} x_{i}^{k}\right)=\left(\prod_{k=1}^{n} d^{4} x_{i}^{k} 1_{\mathscr{F}\left(x_{i}^{k-1}\right)}\left(x_{i}^{k}\right)\right) \times \\
& \quad \times \frac{1}{(c \tau)^{n}} \exp \left(-\frac{1}{c \tau} \sum_{k=1}^{n} \mathrm{t}-\operatorname{dist}\left(x_{i}^{k}, x_{i}^{k-1}\right)\right) \hat{\jmath}_{i}^{1} \hat{\jmath}_{i}^{2} \cdots \hat{\jmath}_{i}^{n} \hat{\jmath}_{i}^{n} \cdots \hat{\jmath}_{i}^{2} \hat{\jmath}_{i}^{1}
\end{aligned}
$$

with $1_{B}$ the indicator function of the set $B$ and $\hat{j}_{i}^{k}$ the self-adjoint "collapse" operator defined on $L^{2}\left(\Sigma_{i}^{0}\right)$ as follows. Let, for any spacelike surface $\Sigma$ and $x \in \Sigma, \hat{\jmath}_{\Sigma}(x)$ be the multiplication operator on $L^{2}(\Sigma)$ that multiplies by the function $j_{\Sigma}(x, \cdot)$ defined in (21). Then

$$
\hat{\jmath}_{i}^{k}=\hat{U}_{\mathscr{H}\left(x_{i}^{k}, x_{i}^{k-1}\right)}^{\Sigma_{0}^{0}} \hat{\jmath}_{\mathscr{H}\left(x_{i}^{k}, x_{i}^{k-1}\right)}\left(x_{i}^{k}\right) \hat{U}_{\Sigma_{i}^{0}}^{\mathscr{H}\left(x_{i}^{k}, x_{i}^{k-1}\right)} .
$$

In our notation $E_{i, x}^{(n)}$ we conceal the (uninteresting) dependence on the choice of $\Sigma_{i}^{0}$. Normalization of $E_{i}^{(n)}$, i.e., $E_{i}^{(n)}\left(\mathscr{M}_{i}^{n}\right)=\hat{1}$, can also directly be seen from the fact that

$$
E_{i}^{(n+1)}\left(\prod_{k=1}^{n} d^{4} x_{i}^{k} \times \mathscr{F}\left(x_{i}^{n}\right)\right)=E_{i}^{(n)}\left(\prod_{k=1}^{n} d^{4} x_{i}^{k}\right)
$$


which in turn follows from

$$
\int_{\mathscr{F}(x)} d^{4} y \frac{1}{c \tau} \exp \left(-\frac{1}{c \tau} \mathrm{t} \text {-dist }(y, x)\right) \hat{\jmath}_{\mathscr{H}(y, x)}(y)^{2}=\hat{1} .
$$

Eq. (27) is, in effect, a Heisenberg picture formulation, as illustrated particularly by the way the unitary operators occur in (30), and by the arbitrariness of the surfaces $\Sigma_{i}^{0}$, which may even lie in the future of (some of) the flashes.

\subsection{The Flashes up to Given Surfaces}

It is a corollary of (27) that the probability for obtaining, up to spacelike surfaces $\Sigma_{i}$, a particular sequence $x_{i}^{1}, \ldots, x_{i}^{n_{i}}$ of flashes for every type $i$, is

$$
\begin{array}{r}
\operatorname{Prob}\left(Q_{i} \cap \mathscr{P}\left(\Sigma_{i}\right) \in\left\{x_{i}^{0}\right\} \times d^{4} x_{i}^{1} \times \ldots \times d^{4} x_{i}^{n_{i}} \forall i\right)= \\
=\left\langle\Psi^{0}\left|E^{(\vec{n}+1)}\left(\prod_{i} d^{4} x_{i}^{1} \times \cdots \times d^{4} x_{i}^{n_{i}} \times \mathscr{F}\left(\Sigma_{i}\right)\right)\right| \Psi^{0}\right\rangle
\end{array}
$$

with $\vec{n}+1:=\left(n_{1}+1, \ldots, n_{N}+1\right)$.

\subsection{Flash Rate in the Temporal Picture}

It is often desirable to select a time coordinate - a slicing parametrized by $t$ into spacelike surfaces $\Sigma_{i}(t)$-in each $\mathscr{M}_{i}$ and to employ a picture in which everything evolves as a function of the time coordinate. In this picture, the interesting quantity is the probability of having a flash of type ${ }^{4} I \in\{1, \ldots, N\}$ between $t$ and $t+d t$, conditional on the flashes up to time $t$. For this we obtain the following formula, in which we assume that the given flashes are timelike separated, $x_{i}^{k} \in \mathscr{F}\left(x_{i}^{k-1}\right)$ for all $i$ and $k \leq n_{i}$, and that $d^{3} y$ is a volume element in $\Sigma_{I}(t)$. We denote by $d t \times d^{3} y$ the 4 -volume element between $\Sigma_{I}(t)$ and $\Sigma_{I}(t+d t)$ swept out by the normals on $\Sigma_{I}(t)$ over $d^{3} y$; the volume of this element is $c(y) d t d^{3} y$ with $c(y) d t=\mathrm{t}$-dist $\left(\Sigma_{I}(t+d t), y\right)$.

$$
\begin{aligned}
\operatorname{Prob} & \left(Q_{I} \cap \mathscr{F}\left(\Sigma_{I}(t)\right) \cap \mathscr{P}\left(\Sigma_{I}(t+d t)\right)=\{Y\}, Y \in d t \times d^{3} y \mid\right. \\
& \left.Q_{i} \cap \mathscr{P}\left(\Sigma_{i}(t)\right)=\left\{x_{i}^{0}, \ldots, x_{i}^{n_{i}}\right\} \forall i\right)= \\
= & c(y) d t d^{3} y 1_{\mathscr{F}\left(x_{I}^{n_{I}}\right)}(y) \frac{1}{c \tau} \exp \left(-\frac{1}{c \tau} \operatorname{t}-\operatorname{dist}\left(y, x_{I}^{n_{I}}\right)\right) \times \\
& \times \frac{\left\langle\Psi_{t}\left|E_{1, x_{1}^{n_{1}}}^{(1)}\left(\mathscr{F}\left(\Sigma_{1}(t)\right)\right) \otimes \cdots \otimes \hat{\jmath}_{I}(y)^{2} \otimes \cdots \otimes E_{N, x_{N}^{n_{N}}}^{(1)}\left(\mathscr{F}\left(\Sigma_{N}(t)\right)\right)\right| \Psi_{t}\right\rangle}{\left\langle\Psi_{t}\left|\otimes_{i} E_{i, x_{i}^{n_{i}}}^{(1)}\left(\mathscr{F}\left(\Sigma_{i}(t)\right)\right)\right| \Psi_{t}\right\rangle}
\end{aligned}
$$

\footnotetext{
${ }^{4}$ Unlike as usual, the capitalization is not meant here to indicate that $I$ is a random variable, but merely to distinguish that particular number from the other $i$ 's.
} 
where

$$
\Psi_{t}=\gamma_{t}\left(\bigotimes_{i} \hat{j}_{i}^{n_{i}} \cdots \hat{\jmath}_{i}^{1}\right) \Psi^{0}
$$

with $\left(\operatorname{arbitrary}^{5}\right)$ normalization factor $\gamma_{t}$, and $\hat{\jmath}_{I}(y)$ (appearing in the $I$-th factor of the tensor product) is the collapse operator corresponding to a flash at $y$,

$$
\hat{\jmath}_{I}(y)=\hat{U}_{\mathscr{H}\left(y, x_{I}^{n_{I}}\right)}^{\Sigma^{0}} \hat{\jmath}_{\mathscr{H}\left(y, x_{I}^{n_{I}}\right)}(y) \hat{U}_{\Sigma_{I}^{0}}^{\mathscr{H}\left(y, x_{I}^{n_{I}}\right)}
$$

\section{The Temporal Picture}

We now describe the model in the temporal picture, based on a slicing of space-time. This is basically a discussion of (34), the flash rate formula of the temporal picture.

With every time $t$ we associate a wavefunction

$$
\Psi_{t}=\gamma_{t}\left(\bigotimes_{i} \hat{U}_{\Sigma_{i}^{0}}^{\Sigma_{i}(t)} \hat{\jmath}_{i}^{n_{i}} \cdots \hat{\jmath}_{i}^{1}\right) \Psi^{0}
$$

the same as in (35) except that we now shift from the Heisenberg to the Schrödinger picture and use the unitary Dirac evolution to define $\Psi_{t}$ on $\Sigma_{1}(t) \times \cdots \times \Sigma_{N}(t)$. $\Psi_{t}$ evolves unitarily apart from collapses. We remark that $\Psi_{t}$ in fact depends only on the surface at time $t$ : it depends on the slicing of space-time only through this surface, in the sense that different slicings that happen to coincide at $t$ have the same $\Psi_{t}$. As is clear from (35), $\Psi_{t}$ is determined by the initial wavefunction $\Psi^{0}$ and the flashes up to time $t$.

Whenever a flash occurs, the wavefunction $\Psi_{t}$ gets collapsed by applying to it the suitable collapse operator $\hat{j}_{i}^{n_{i}+1}$ (and then renormalizing). Recall that also in the GRW model, the wavefunction gets collapsed by applying the appropriate collapse operator. However, while in the GRW model the collapse operator is always a multiplication operator in the position representation, this is not necessarily the case in our model; instead, $\hat{\jmath}_{i}^{n_{i}+1}$ arises from a multiplication operator on the suitable hyperboloid, $\mathscr{H}\left(X_{i}^{n_{i}+1}, X_{i}^{n_{i}}\right)$, by using the Dirac propagators to get to $L^{2}\left(\mathscr{H}\left(X_{i}^{n_{i}+1}, X_{i}^{n_{i}}\right)\right)$ and back; in other words, $\hat{J}_{i}^{n_{i}+1}$ is a multiplication operator evolved, as in the Heisenberg picture, to another surface.

To compute the flash rate, it is unnecessary to know the entire history of collapses. Instead, it suffices to know the present wavefunction $\Psi_{t}$ and the last collapses of all types, $X_{i}^{n_{i}}$. In other words, the evolution of the $(N+1)$-tuple $\left(\Psi_{t}, X_{1}^{n_{1}}, \ldots, X_{N}^{n_{N}}\right)$ is a Markov process. Although this is true as well in the GRW model, there, by way of contrast, already $\Psi_{t}$ itself follows a Markov process, and knowledge about the space-time locations of the previous flashes does not add any information, beyond what is encoded in $\Psi_{t}$, about the distribution of the future flashes.

\footnotetext{
${ }^{5}$ That is, although we will consider the usual normalization $\left\langle\Psi_{t} \mid \Psi_{t}\right\rangle=1$, (34) holds for any choice of $\gamma_{t}$, for instance $\gamma_{t}=1$. Another useful possibility is to choose $\gamma_{t}$ such that the denominator in the last line of (34) becomes 1 .
} 
Another difference between the flash rate formula of our model (34) and that of the GRW model (6) is the appearance of a denominator and an further factors, $E_{i}^{(1)}\left(\mathscr{F}\left(\Sigma_{i}(t)\right)\right)$, in the numerator; they are needed to correct the rates for conditioning on the knowledge that no further collapses have occured until time $t$.

How does the wavefunction transform under a change of slicing of space-time, such as given by a Lorentz boost in Minkowski space-time? In two ways. First, some flashes may lie in the future of the new surface $\Sigma_{i}^{\prime}(t)$ but in the past of the old surface $\Sigma_{i}(t)$ and vice versa; consequently, the corresponding wavefunctions differ by application of the collapse operators (respectively their inverses, and renormalization) belonging to these flashes. Second, on top of that the wavefunctions differ by the unitary Dirac propagator from one surface to the other.

\section{The Low Velocity Regime}

In this section we consider Minkowski space-time and assume the existence of a Lorentz frame in which all velocities, at which wave packets move or spread, are small compared to the speed of light. We point out that in this regime, and in this frame, our model approaches the GRW model.

The basic observation is that for large $t$, such as $t$ of the order of magnitude of $\tau \approx 10^{15}$ sec, the hyperboloid $\mathscr{H}_{c t}$ becomes rather flat and is well approximated by the plane $x^{0}=c t$ tangent to the hyperboloid, at least in the neighborhood of their common point $(c t, 0,0,0)$ with radius $R$ such that $R \ll c t$, or $R \ll 10^{8}$ light-years. As a consequence, to multiply by a Gaussian with width $a \ll R$ centered somewhere inside this neighborhood means approximately the same on the hyperboloid and on the plane. And since, by the assumption on velocities, the wavefunction will lie almost completely within this neighborhood for $t$ of the order of magnitude of $\tau$, the action of the collapse operator agrees approximately with that of the GRW model. Similarly, the factor $1_{\mathscr{F}(X)}$ is 1 on the support of $\Psi_{t}$.

Replacing our collapse operators with those of GRW, we obtain $E_{i}^{(1)}\left(\mathscr{F}\left(\Sigma_{i}(t)\right)\right) \approx$ $e^{-\left(t-T_{i}\right) / \tau} \hat{1}$, so that, since $c(y)=c$ for this slicing, (34) in fact reduces to (6).

There is a subtlety in that if $N$ is very large, flashes do sometimes occur for which the timelike distance $c \Delta T$ from the previous flash of the same type is not of the order of magnitude of $c \tau$, but much smaller. (However, $c \Delta T$ is still much larger than $a$ except in very rare cases that occur only once in $c \tau / a \approx 10^{30}$ collapses.) Fortunately, relevant wave functions obey a bound on their spread: it is at most $a$ initially (thanks to the previous collapse) and grows much slower than at rate $c$. Thus, the spread is still much smaller than $c \Delta T$, so that the collapse operators of the two models do not differ appreciably on these wavefunctions. 


\section{Predictions}

What predictions does the model entail? To what extent are they in agreement with quantum mechanics? In what way do they deviate from the predictions of the nonrelativistic GRW model?

To begin with, a difficulty with obtaining any predictions at all from the model is that it does not involve any interaction, and thus does not support the formation of macroscopic bodies such as observers or apparatuses. However, we can say what the predictions will be like once interaction is included in whatever way, be it by particle creation and annihilation or by a modification of the unitary propagators: we can consider a wavefunction as would arise from interaction.

As a corollary of the previous section, for any experiment in which no parts need move at relativistic speeds our model approximates the GRW model and is thus in agreement with quantum mechanics to the same extent as the GRW model; this includes all presently doable experiments; for a discussion of future experiments that may distinguish spontaneous collapse theories from quantum mechanics, see Section V of [3]. The model is in particular in agreement with the result of EPR-Bell experiments such as Aspect's [2] and thus violates Bell's locality inequality [5].

A general pattern of behavior of the model follows from another trait it has in common with the GRW model: that disentangled subsystems are governed by the same laws as the whole, and follow an independent collapse process. Therefore, a small system of $N_{1}<10^{5}$ particles will not collapse for the next thousand years, provided it stays disentangled. A macroscopic body, however, cannot support superpositions over distances much wider than $a \approx 10^{-7} \mathrm{~m}$ for longer than a split second.

The most obvious deviation of our model from the GRW model is that a system moving at a speed close to $c$ will have a reduced rate of spontaneous collapse, reduced by just the factor that one would expect from a naive application of time dilation. This will be hard to see in experiment, of course, given that as yet we cannot see any spontaneous collapses in experiment.

Extensions of the model to quantum field theory might deviate from the nonrelativistic GRW model in a prediction of spontaneous pair creation at the flashes, as discussed in Section 3.7 .

Superluminal communication on the basis of entangled sets of particles is impossible in our model. To see this, suppose that Alice and Bob are widely separated and share a system of entangled particles; the marginal distribution of the $Q_{i}$ for the $i$ 's of all particles located on Alice's side is, as follows from (27) and (29), independent of the fields applied to the $x_{i}$ for the $i$ 's of the particles on Bob's side, such as the metric and electromagnetic vector potential of $\mathscr{M}_{i}$.

\section{Perspective}

Our model seems to be the first model in the literature that achieves all of the following:

(i) it describes a possible (many-particle) world in which outcomes of experiments 
have (to a sufficient degree of accuracy for all cases presently testable) the probabilities prescribed by quantum theory,

(ii) it describes objective events in space-time (the flashes), in contrast to theories merely associating a wavefunction with every spacelike surface,

(iii) it is fully compatible with relativity in that it does not rely on a preferred slicing of space-time, and

(iv) it works in the continuum, in contrast to theories assuming a discrete space-time.

We give a brief overview of the literature concerning relativistic models explaining the probabilities prescribed by quantum theory. Such models come in two varieties, either as a variant of Bohmian mechanics or as a spontaneous collapse theory.

Among the variants of Bohmian mechanics, Bohm [8] gives a Lorentz-invariant equation of motion for a single Dirac particle; Bohm and Hiley [9] give a many-particle version based on a preferred Lorentz frame; Dürr et al. [16] generalize to an arbitrary spacelike preferred slicing of space-time, possibly determined by a covariant law involving the wavefunction; Samols [26, 27] gives a Bohm-type model on a discrete space-time using a preferred slicing; Goldstein and Tumulka 20] give a nonlocal many-particle version without preferred slicing, which however fails to yield any probabilities. Both Berndl et al. [7] and Dewdney and Horton [10] suggest, instead of a preferred slicing, a preferred joint parametrization (or synchronization) of the world lines, and thus obtain a nonlocal Bohm-type dynamics; however, this does not really conform any better with the spirit of relativity than a preferred slicing; in addition, the models fail to yield any probabilities.

All spontaneous collapse models deviate slightly from the quantum prescriptions in their probabilities. An overview of spontaneous collapse models is given by Bassi and Ghirardi in [3]. Dove and Squires [12, 13] have made steps towards a relativistic model based on discrete flashes. ${ }^{6}$ Dowker and coworkers [14, 15] give a collapse model on a discrete space-time that does not need a preferred slicing; it is not known how this model could be adapted to a continuum. All other efforts towards a relativistic collapse model are based on the approach of continuous spontaneous localization (CSL), corresponding to diffusion processes in Hilbert space; some references describing research in this direction are [24, 18, 11, 25, 23].

A somewhat surprising feature of the present situation is that we seem to arrive at the following alternative: Bohmian mechanics shows that one can explain quantum mechanics, exactly and completely, if one is willing to pay the price of using a preferred slicing of space-time; our model suggests that one should be able to avoid a preferred slicing if one is willing to pay the price of a certain deviation from quantum mechanics.

\footnotetext{
${ }^{6}$ Their paper 13 (reprinted, with minor extensions, as Chapter 7 of 12 ) is irritating in that the authors claim to provide a Lorentz-invariant collapse model but do not keep their promise. They define an evolution of the wavefunction given the flashes, according to which the wavefunction collapses on the future light cone of each flash and evolves unitarily in between. However, they do not specify a probability law for the flashes.
} 
Note added. Several articles discussing the relativistic model of this paper have been written since its first preprint version became available in 2004 [22, 29, 1].

Acknowledgments. I wish to thank Fay Dowker, Detlef Dürr, Shelly Goldstein, Philip Pearle, and Nino Zanghì for their critical comments on a draft. I have also profited a lot from discussions with each of them and, in addition, with GianCarlo Ghirardi and Peter Pickl. This work was supported in part by INFN.

\section{References}

[1] Allori V., Goldstein S., Tumulka R., and Zanghì, N.: "On the Common Structure of Bohmian Mechanics and the Ghirardi-Rimini-Weber Theory", quant-ph/0603027

[2] Aspect, A., Dalibard, J., Roger, G.: "Experimental test of Bell's inequalities using time-varying analyzers", Phys. Rev. Lett. 49, 1804-1807 (1982)

[3] Bassi, A., Ghirardi, G.C.: "Dynamical Reduction Models", Phys. Rep. 379, 257427 (2003) and quant-ph/0302164

[4] Bell, J. S.: "Are there quantum jumps?", in Schrödinger. Centenary celebration of a polymath, C. W. Kilmister (ed.), p. 41-52. Cambridge: Cambridge University Press (1987). Reprinted in [5, p. 201-212.

[5] Bell, J. S.: Speakable and unspeakable in quantum mechanics. Cambridge: Cambridge University Press (1987)

[6] Bell, J. S.: "Towards An Exact Quantum Mechanics", in Themes in contemporary physics, II, S. Deser and R. J. Finkelstein (eds.), p. 1-26. Teaneck, NJ: World Scientific (1989)

[7] Berndl, K., Dürr, D., Goldstein, S., Zanghì, N.: "Nonlocality, Lorentz Invariance, and Bohmian Quantum Theory", Phys. Rev. A 53, 2062-2073 (1996) and quant-ph/9510027

[8] Bohm, D.: "Comments on an Article of Takabayasi concerning the Formulation of Quantum Mechanics with Classical Pictures", Progr. Theoret. Phys. 9, 273-287 (1953)

[9] Bohm, D., Hiley, B.J.: The Undivided Universe: An Ontological Interpretation of Quantum Theory. London: Routledge (1993)

[10] Dewdney, C., Horton, G.: "A non-local, Lorentz-invariant, hidden-variable interpretation of relativistic quantum mechanics based on particle trajectories", J. Phys. A: Math. Gen. 34, 9871-9878 (2001) and quant-ph/0110007 
[11] Diósi, L.: "Relativistic theory for continuous measurement of quantum fields", Phys. Rev. A 42, 5086-5092 (1990)

[12] Dove, C.: "Explicit Wavefunction Collapse and Quantum Measurement", Ph.D. thesis, Department of Mathematical Sciences, University of Durham (1996)

[13] Dove, C., Squires, E. J.: "A Local Model of Explicit Wavefunction Collapse", quant-ph/9605047

[14] Dowker, F., Henson, J.: "Spontaneous Collapse Models on a Lattice", J. Statist. Phys. 115, 1327-1339 (2004) and quant-ph/0209051

[15] Dowker, F., Herbauts, I.: "Simulating causal collapse models", quant-ph/0401075

[16] Dürr, D., Goldstein, S., Münch-Berndl, K., Zanghì, N.: "Hypersurface BohmDirac models", Phys. Rev. A 60, 2729-2736 (1999) and quant-ph/9801070

[17] Dürr, D., Pickl, P.: "Flux-Across-Surfaces Theorem for a Dirac Particle", J. Math. Phys. 44, 423-456 (2003) and math-ph/0207010

[18] Ghirardi, G.C., Grassi, R., Pearle, P.: "Relativistic dynamical reduction models: general framework and examples", Found. Phys. 20, 1271-1316 (1990)

[19] Ghirardi, G.C., Rimini, A., Weber, T.: "Unified dynamics for microscopic and macroscopic systems", Phys. Rev. D 34, 470-491 (1986)

[20] Goldstein, S., Tumulka, R.: "Opposite arrows of time can reconcile relativity and nonlocality", Class. Quantum Gravity 20, 557-564 (2003) and quant-ph/0105040

[21] Kent, A.: "Quantum jumps" and indistinguishability", Modern Phys. Lett. A 4(19), 1839-1845 (1989)

[22] Maudlin, T.: "Non-Local Correlations in Quantum Theory: Some Ways the Trick Might Be Done", to appear in Einstein, Relativity, and Absolute Simultaneity, Q. Smith and W. L. Craig (ed.), London: Routledge (2007).

[23] Nicrosini, O., Rimini, A.: "Relativistic spontaneous localization: a proposal", Found. Phys. 33, 1061-1084 (2003) and quant-ph/0207145

[24] Pearle, P.: "Toward a Relativistic Theory of Statevector Reduction", in SixtyTwo Years of Uncertainty: Historical, Philosophical, and Physical Inquiries into the Foundations of Quantum Physics, A.I. Miller (ed.), volume 226 of NATO ASI Series B, p. 193-214. New York: Plenum Press (1990)

[25] Pearle, P.: "Relativistic Collapse Model With Tachyonic Features", Phys. Rev. A 59, 80-101 (1999) and quant-ph/9902046

[26] Samols, T. M.: "A stochastic model of a quantum field theory", J. Statist. Phys. 80, 793-809 (1995) and hep-th/9501117 
[27] Samols, T. M.: "A realistic formulation of quantum field theory", in Bohmian mechanics and quantum theory: an appraisal, J. Cushing, A. Fine, and S. Goldstein (eds.), volume 184 of Boston Stud. Philos. Sci., pages 191-196. Dordrecht: Kluwer Acad. Publ. (1996)

[28] Teufel, S., Tumulka, R.: "Simple Proof for Global Existence of Bohmian Trajectories", Commun. Math. Phys. 258, 349-365 (2005) and math-ph/0406030

[29] Tumulka, R.: "Collapse and Relativity", in Quantum Mechanics: Are there Quantum Jumps? and On the Present Status of Quantum Mechanics, A. Bassi, D. Dürr, T. Weber and N. Zanghì (eds.), AIP Conference Proceedings 844, 340-352. American Institute of Physics (2006) and quant-ph/0602208 